\section{Kidney \\ Blood Pressure Research}

Original Paper

\title{
Identification of Genes and Pathways Associated with Kidney Ischemia-Reper- fusion Injury by Bioinformatics Analyses
}

\author{
Wei Feng Rongwei Tang Xudong Ye Chao Xue Yunhua Liao \\ Department of Nephrology, The First Affiliated Hospital of Guangxi Medical University, Nanning, China
}

\section{Key Words}

Kidney ischemia-reperfusion injury • Differentially expressed genes • Functional enrichment analysis • Protein-protein interaction

\begin{abstract}
Background/Aims: Ischemia-reperfusion (IR) injury in the kidney is a major cause of acute kidney injury in humans. However, the molecular mechanisms responsible for the progression of kidney IR injury still need to be explored. In this study, we aimed to explore the underlying genes and pathways associated with kidney IR injury. Methods: Gene microarray of GSE27274 was downloaded from the Gene Expression Omnibus database. The differentially expressed genes (DEGs) between kidney IR injury and kidney IR rat samples were analyzed. Gene Ontology biological process (BP) and pathway enrichment analyses of DEGs were performed, followed by protein-protein interaction (PPI) network construction. Results: A total of 88 upregulated and 102 down-regulated DEGs were identified. The up-regulated DEGs including FK506 binding protein 1A (Fkb1a) were mainly enriched in biological processes (BPs) related to protein ubiquitination. The down-regulated DEGs including complement component 5 (C5) were enriched in complement and coagulation cascades pathway. Choline phosphotransferase 1 (Chpt1) was enriched in glycerophospholipid metabolism pathway. In the PPI network, heme oxygenase (decycling) 1 (Hmox1) was as a hub gene that interacted with the maximum nodes. Conclusions: DEGs of Fkb1a, C5, Chpt1, and Hmox1, as well as complement and coagulation cascades pathway, glycerophospholipid metabolism pathway, and BP terms related to protein ubiquitinatione may be the potential targets for diagnosis and treatment of kidney IR injury.
\end{abstract}

Copyright @ 2016 S. Karger AG, Basel

\section{Introduction}

Kidney disease is a major public health concern with significantly increased morbidity and mortality [1]. Ischemia-reperfusion (IR) injury in the kidney is a major cause of acute kidney injury in humans [2]. This injury occurs when blood flow is restored after an extended 


\section{Kidney Blood Pressure Research}

period of ischemia [3]. It is a common source of the incidence and death rates in stroke, myocardial infarction and gut ischemia. Currently, there is often no specific therapy for IR injury [4].

The pathophysiology of IR injury is complex, including molecular oxygen, neutrophils, and components of the activated complement cascade $[5,6]$. Recent studies have found that cytokines, adhesion molecules, platelet-activating factors, endothelin and leukotrienes play important roles in the pathophysiology of IR injury $[7,8]$. Importantly, genome-wide expression analysis of the kidney after kidney injury caused by ischemic injury has revealed that interleukin 1 beta and intercellular adhesion molecule 1 are up-regulated in kidney IR injury [9]. Additionally, kidney injury molecule-1 mRNA levels have been found elevated on the apical membrane of proximal tubular epithelial cells in most injured regions of the kidney [10]. Based on the dataset of GSE9943, Shen et al. [11] revealed that miR-29c and transient receptor potential canonical (TRPC6) were potential novel targets for IR injury using bioinformatics approach. Although significant progress has been made to understand the potential significance of genetic factors in the kidney IR injury, relatively little is known about the molecular mechanisms responsible for the development and progression of kidney IR injury.

In this study, we downloaded the gene expression profile GSE27274 which was provided by Krishnamoorthy et al. [2]. Several studies have performed bioinformatics approach to study the molecular mechanisms of kidney IR injury based on this expression profile data, however, most of them only performed differentially expressed genes (DEGs) analysis or pathway analysis $[12,13]$. At present, we not only identified the differentially expressed genes (DEGs) between kidney IR injury and kidney IR samples, but also performed functional enrichment analyses and protein-protein interaction (PPI) networks analysis for the identified DEGs to further explore their functions in kidney IR injury. Findings of this study may potentially serve as biomarkers in both diagnosis and treatment of kidney IR injury.

\section{Materials and Methods}

\section{Affymetrix microarray data}

The gene expression profile data of GSE27274 were downloaded from Gene Expression Omnibus (http:// www.ncbi.nlm.nih.gov/geo/) database based on the platform of Illumina ratRef-12 v1.0 expression beadchip. The dataset contains 24 samples and samples of GSM674258, GSM674259, GSM674260, GSM674264, GSM674265 and GSM674266 were analyzed in this study.

Data preprocessing and differential expression analysis

The original array data were downloaded and normalized. The expression values of multiple probes for a given gene were reduced to a single value by taking the average expression value. The limma [14] package in $\mathrm{R}$ language was used to identify the DEGs. The $\log _{2}$-fold change $\left(\log _{2} \mathrm{FC}\right)$ and false discovery rate (FDR) were calculated. $\left|\log _{2} \mathrm{FC}\right|>0.5$ and FDR $<0.05$ were considered as the cutoffs.

\section{Functional enrichment analyses}

The Database for Annotation, Visualization and Integrated Discovery (DAVID, http://david.abcc.ncifcrf.gov/ knowledgebase// [15] is a comprehensive set of functional annotation tools, which has been developed for relating the functional terms with gene lists by clustering algorithm. In this study, we performed Gene Ontology (GO) biological process (BP) and Kyoto Encyclopedia of Genes and Genomes (KEGG) pathway enrichment analyses for the DEGs using the DAVID online tool. The p-value $<0.01$ was set as threshold.

PPI network construction

The Search Tool for the Retrieval of Interacting Genes (STRING, http://string-db.org/) [16] database is designed to evaluate the PPI information. In this study, STRING online tool was applied to analyze the PPI of DEGs and only those experimentally validated interactions with a combined score $>0.4$ were selected as significant interactions. 


\section{Kidney \\ Blood Pressure Research}

Kidney Blood Press Res 2016;41:48-54

DOI: $10.1159 / 000368546$

(C) 2016 S. Karger AG, Basel

www.karger.com/kbr

Table 1. Gene Ontology (GO) functional enrichment analysis for the up- and down-regulated differentially expressed genes (DEGs) (top 5)

\begin{tabular}{|c|c|c|c|}
\hline \multirow{2}{*}{$\begin{array}{l}\text { ID } \\
\text { Up-regulated DEGs }\end{array}$} & \multicolumn{2}{|c|}{ Term } & \multirow[t]{2}{*}{ P-value } \\
\hline & & & \\
\hline GO:0031398 & \multicolumn{2}{|c|}{ positive regulation of protein ubiquitination } & 4.46E-04 \\
\hline GO:0044092 & \multicolumn{2}{|c|}{ negative regulation of molecular function } & $6.75 \mathrm{E}-04$ \\
\hline GO:0031396 & \multicolumn{2}{|c|}{ regulation of protein ubiquitination } & $1.06 \mathrm{E}-03$ \\
\hline GO:0043086 & \multicolumn{2}{|c|}{ negative regulation of catalytic activity } & $1.23 \mathrm{E}-03$ \\
\hline GO:0032269 & \multicolumn{2}{|c|}{ negative regulation of cellular protein metabolic process } & $1.62 \mathrm{E}-03$ \\
\hline \multicolumn{4}{|c|}{ Down-regulated DEGs } \\
\hline GO:0046689 & \multicolumn{2}{|c|}{ response to mercury ion } & $4.66 \mathrm{E}-02$ \\
\hline GO:0008285 & \multicolumn{2}{|c|}{ negative regulation of cell proliferation } & $5.10 \mathrm{E}-02$ \\
\hline GO:0006732 & \multicolumn{2}{|c|}{ coenzyme metabolic process } & $5.27 \mathrm{E}-02$ \\
\hline \multirow{8}{*}{$\begin{array}{l}\text { Table 2. Pathway } \\
\text { e } \mathrm{n} \text { r i c h m e n t } \\
\text { analysis for the } \\
\text { up- and down-re- } \\
\text { gulated differen- } \\
\text { tially expressed } \\
\text { genes (DEGs) }\end{array}$} & Category & Term & P-value \\
\hline & \multicolumn{3}{|c|}{ Up-regulated DEGs } \\
\hline & rno03050 & Proteasome & 0.0387 \\
\hline & rno00670 & One carbon pool by folate & 0.0900 \\
\hline & \multicolumn{3}{|c|}{ Down-regulated DEGs } \\
\hline & rno00564 & Glycerophospholipid metabolism & 0.0356 \\
\hline & rno04610 & Complement and coagulation cascades & 0.0444 \\
\hline & rno00360 & Phenylalanine metabolism & 0.0746 \\
\hline
\end{tabular}

\section{Results}

Identification of DEGS

For the dataset GSE27274, a total of 190 DEGs were obtained after data preprocessing. Among them, 88 were down-regulated and 102 were up-regulated in the renal IR injury group.

\section{Functional enrichment analyses}

The top 5 BP terms enriched by up-regulated and down-regulated DEGs were shown in Table 1. As shown in the table, the up-regulated DEGs were enriched in BP terms related to protein ubiquitination, molecular function, and cellular protein metabolic process, such as FK506 binding protein 1A (Fkb1a) and Proteasome (Prosome, Macropain) 26S Subunit, ATPase, 2 (Psmc2). BP terms enriched by down-regulated DEGs were mainly associated with response to mercury ion, cell proliferation, and coenzyme metabolic process. The up-regulated DEGs were enriched in 2 pathways including proteasome and one carbon pool by folate. The down-regulated DEGs were enriched in 3 pathways including glycerophospholipid metabolism (choline phosphotransferase 1 (Chpt1)), complement and coagulation cascades (complement component 5 (C5)), and phenylalanine metabolism (Table 2).

\section{PPI network construction}

Based on STRING database, the PPI network was constructed (Fig. 1). The figure showed that heme oxygenase (decycling) 1 (Hmox1) was as a hub gene that interacted with the maximum nodes. 


\section{Kidney \\ Blood Pressure Research}

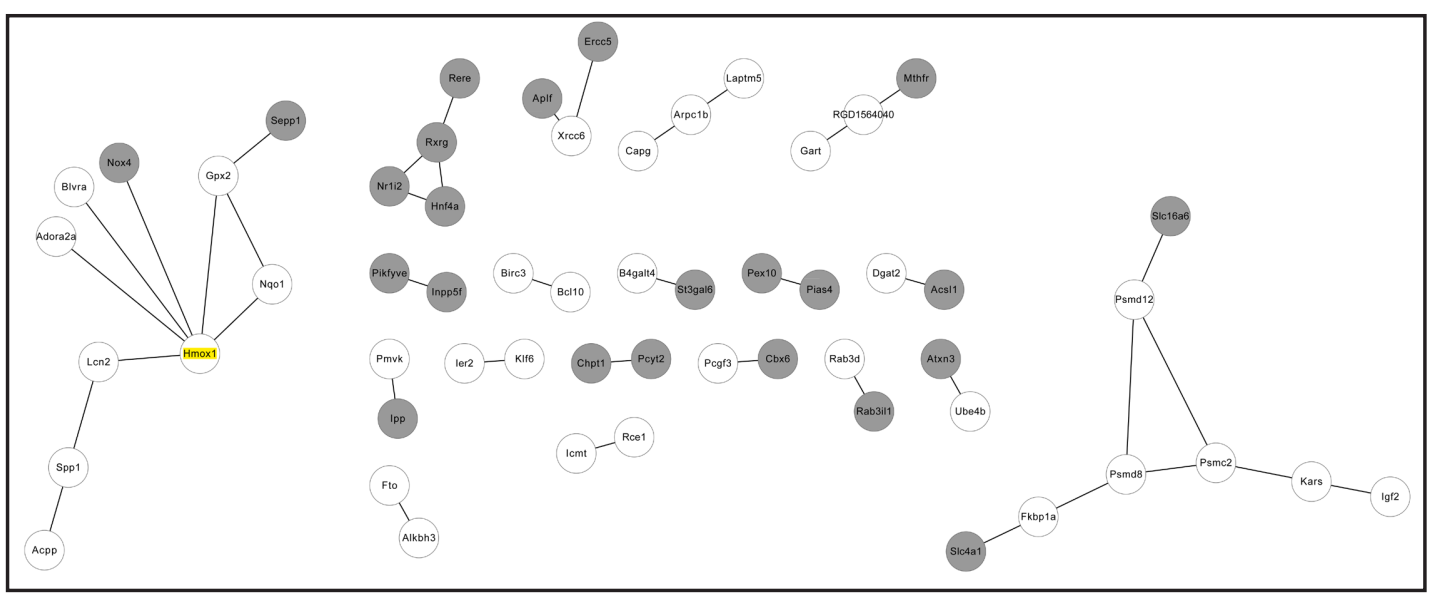

Fig. 1. The protein-protein interaction network of differentially expressed genes (DEGs). White node stands for up-regulated DEGs; gray node stands for down-regulated DEGs.

\section{Discussion}

Our study found that 190 DEGs between kidney IR injury and kidney IR samples were obtained after data processing. The up-regulated DEGs were significantly enriched in BP terms related to protein ubiquitinatione. On the other hand, the down-regulated DEGs were enriched in complement and coagulation cascades pathway, and metabolism related pathways. In addition, in the PPI network, Hmox1 was as a hub gene which interacted with the maximum nodes. The results suggested that these genes and pathways may play important roles in the progression of kidney IR injury.

Studies have suggested that the ubiquitin-proteasome system mediates the degradation of most intracellular proteins in cells, including ubiquitination of protein molecules and degradation of the ubiquitinated protein by the proteasome $[17,18]$. Ubiquitinated proteins usually accumulate in cells when the function of proteasome is insufficient [19]. Tian et al. [17] have found a global increase of ubiquitinated proteins in IR hearts. In the present study, BP terms related to protein ubiquitination were enriched by the up-regulated DEGs including Fkb1a. Fkb1a encodes a member of the immunophilin protein family, which play a role in immunoregulation and basic cellular processes involving protein folding and trafficking [20]. Accumulation and activation of immune system cells in the kidney during IR is a major mediator of kidney injury. Animal models experiments have revealed that both the innate and adaptive immune systems mediate IR injury in the kidney [21]. Importantly, ischemic damage is the most common cause of delayed graft function in accompany with tissue inflammation [22]. Boros and Bromberg [23] also reported that IR injury is a multifactorial antigen-independent inflammatory condition. Therefore, the up-regulation of Fkb1a in kidney IR injury may be associated with the inflammation of this injury.

In addition, the down-regulated DEGs including $C 5$ were found to be enriched in the pathway of complement and coagulation cascades. The complement system, consisting of multiple cascades, is a component of the immune response, which plays an important role in the regulation of the inflammatory response [24]. Study has found that the complement cascade plays a key role in numerous systemic organ IR models of tissue damage [25-27]. Shen et al. [11] have also suggested that complement and coagulation cascades pathway is a significant pathway in IR injury. For the enriched DEG of $C 5$, it has been suggested to play an important role in inflammatory and cell killing processes. Importantly, the generation of C5 has also been implicated as playing a potential role in IR injury models of rat myocardium, rat liver, and baboon myocardium [28-30]. Taken together, the results suggest a potential 


\section{Kidney \\ Blood Pressure Research}

place for complement and coagulation cascades pathway and $C 5$ in the treatment of kidney IR injury.

The main function of kidney is to excrete the metabolic wastes; the loss of kidney function can induce significant changes in various metabolites in the body [31]. Qingqing et al. [32] have reported that renal IR produce strong changes of metabolites in kidney cortex, kidney medulla, and plasma over time. In our study, the down-regulated DEGs were also enriched in metabolism related pathways. For instance, Chpt1 was enriched in glycerophospholipid metabolism, which was in accordance with the findings of Cadenas et al. [33], that was, the down-regulation of Chpt1 was responsible for glycerophospholipid and cholesterol metabolism. Therefore, we speculated that the changes of metabolism related pathways in kidney IR injury might be due to the down-regulation of Chpt1.

Furthermore, in the PPI network, Hmox1 was as a hub gene which interacted with the maximum nodes. Hmox1 is a protective gene with anti-inflammatory and anti-apoptotic actions [34]. Hmox1 induction protects cellular against injury caused by the reactive oxygen species (ROS) [35]. The pathophysiology of IR injury includes oxidant stress that contribute to various degrees to the overall organ damage. Formation of ROS has been demonstrated to be the most invoked disease mechanisms in IR injury [36]. Thus, Hmox1 is able to restore tissue architecture and organ function after injury. Many studies have demonstrated its role in IR injury in organs including kidney [37]. Interestingly, in the PPI network, Hmox1 interacted with NAD(P)H dehydrogenase, quinone 1 (Nqo1) which was also an antioxidant protein that regulated ROS generation [38]. Importantly, Gang et al. [39] have suggested that Nqo1 has a protective role against renal injury induced by IR. Therefore, Hmox1 and Nqo1 may play crucial roles in IR injury of kidney.

\section{Conclusion}

Our data provided a comprehensive bioinformatics analysis of DEGs and pathways which might be involved in kidney IR injury. DEGs such as Fkb1a, C5, Chpt1, Hmox1 and Nqo1, as well as complement and coagulation cascades pathway, glycerophospholipid metabolism pathway, and BP terms related to protein ubiquitinatione have the potential to be used as targets for the diagnosis and treatment of kidney IR injury. The findings in current study may contribute to our understanding of the underlying molecular mechanisms of this disorder.

However, this study had some limitations. Firstly, the sample size for microarray analysis was small which might cause a high rate of false positive results. Secondly, this study lacked experimental verification. Therefore further genetic and experimental studies with large sample sizes should be considered to confirm this findings.

\section{Disclosure Statement}

The authors declare no competing interests.

\section{Acknowledgments}

This study was supported by Guangxi administration of traditional Chinese medicine self funded research project (gzzc1228). 


\section{Kidney \\ Blood Pressure Research}

Kidney Blood Press Res 2016;41:48-54

\begin{tabular}{l|l}
\hline DOI: $10.1159 / 000368546$ & (C) 2016 S. Karger AG, Basel
\end{tabular}

Published online: February 04, 2016

www.karger.com/kbr

\section{References}

1 Eckardt K-U, Kasiske BL: Kidney disease: improving global outcomes. Nat Rev Nephrol 2009;5:650-657.

2 Krishnamoorthy A, Ajay AK, Hoffmann D, Kim T-M, Ramirez V, Campanholle G, Bobadilla NA, Waikar SS, Vaidya VS: Fibrinogen $\beta$-derived B $\beta 15-42$ peptide protects against kidney ischemia/reperfusion injury. Blood 2011;118:1934-1942.

3 Hearse DJ, Bolli R: Reperfusion induced injury: manifestations, mechanisms, and clinical relevance. Cardiovasc Res 1992;26:101-108.

4 Zhou W, Farrar CA, Abe K, Pratt JR, Marsh JE, Wang Y, Stahl GL, Sacks SH: Predominant role for C5b-9 in renal ischemia/reperfusion injury. J Clin Invest 2000;105:1363.

5 Korthuis RJ, Smith JK, Carden DL: Hypoxic reperfusion attenuates postischemic microvascular injury. Am J Physiol Heart Circ Physiol 1989;256:H315-H319.

6 Williams FM: Neutrophils and myocardial reperfusion injury. Pharmacol Ther 1996;72:1-12.

7 Rabb H, O'Meara YM, Maderna P, Coleman P, Brady HR: Leukocytes, cell adhesion molecules and ischemic acute renal failure. Kidney Int 1997;51:1463-1468.

8 Daemen MA, Wolfs TG, Buurman WA: Ischemia/reperfusion-induced IFN- $\gamma$ up-regulation: involvement of IL-12 and IL-18. J Immunol 1999;162:5506-5510.

9 Shokeir AA, Barakat N, Hussein AM, Awadalla A, Harraz A, Khater S, Hemmaid K, Kamal AI: Activation of Nrf2 by ischemic preconditioning and sulforaphane in renal ischemia/reperfusion injury: a comparative experimental study. Physiol Res 2015;64:313-323.

10 Vaidya VS, Ramirez V, Ichimura T, Bobadilla NA, Bonventre JV: Urinary kidney injury molecule-1: a sensitive quantitative biomarker for early detection of kidney tubular injury. Am J Physiol Heart Circ Physiol 2006;290:F517-F529.

11 Shen B, Zhou S, He Y, Zhao H, Mei M, Wu X: Revealing the Underlying Mechanism of Ischemia Reperfusion Injury Using Bioinformatics Approach. Kidney Blood Press Res 2013;38:99-108.

12 Ajay AK, Kim T-M, Ramirez-Gonzalez V, Park PJ, Frank DA, Vaidya VS: A bioinformatics approach identifies signal transducer and activator of transcription-3 and checkpoint kinase 1 as upstream regulators of kidney injury molecule-1 after kidney injury. J Am Soc Nephrol 2014;25:105-118.

13 Kim T-M, Ramírez V, Barrera-Chimal J, Bobadilla NA, Park PJ, Vaidya VS: Gene expression analysis reveals the cell cycle and kinetochore genes participating in ischemia reperfusion injury and early development in kidney. Plos One 2011;6:e25679.

14 Smyth GK: Limma: linear models for microarray data; In: Bioinformatics and computational biology solutions using R and Bioconductor. Springer Verlag, New York, 2005, pp. 397-420.

15 Alvord G, Roayaei J, Stephens R, Baseler MW, Lane HC, Lempicki RA: The DAVID Gene Functional Classification Tool: a novel biological module-centric algorithm to functionally analyze large gene lists. Genome Biol 2007;8:R183.

16 Von Mering C, Huynen M, Jaeggi D, Schmidt S, Bork P, Snel B: STRING: a database of predicted functional associations between proteins. Nucleic Acids Res 2003;31:258-261.

17 Tian Z, Zheng H, Li J, Li Y, Su H, Wang X: Genetically induced moderate inhibition of the proteasome in cardiomyocytes exacerbates myocardial ischemia-reperfusion injury in mice. Circ Res 2012;111:532-542.

18 Scruggs SB, Ping P, Zong C: Heterogeneous cardiac proteasomes: mandated by diverse substrates? Physiology 2011;26:106-114.

19 Li J, Horak KM, Su H, Sanbe A, Robbins J, Wang X: Enhancement of proteasomal function protects against cardiac proteinopathy and ischemia/reperfusion injury in mice. J Clin Invest 2011;121:3689.

20 Zhang Y-Y, Shen W, Zhang L-C, Pan Z-Y, Long C-L, Cui W-Y, Zhang Y-F, Wang H: Proteomics reveals potential non-neuronal cholinergic receptor-effectors in endothelial cells. Acta Pharmacol Sin 2014;35:1137-1149.

21 Kinsey GR, Sharma R, Huang L, Li L, Vergis AL, Ye H, Ju S-T, Okusa MD: Regulatory T cells suppress innate immunity in kidney ischemia-reperfusion injury. J Am Soc Nephrol 2009;20:1744-1753.

22 Linkermann A, Bräsen JH, Darding M, Jin MK, Sanz AB, Heller J-O, De Zen F, Weinlich R, Ortiz A, Walczak H: Two independent pathways of regulated necrosis mediate ischemia-reperfusion injury. Proc Natl Acad Sci USA 2013;110:12024-12029.

23 Boros P, Bromberg J: New cellular and molecular immune pathways in ischemia/reperfusion injury. Am J Transplant 2006;6:652-658. 


\section{Kidney \\ Blood Pressure Research}

\begin{tabular}{l|l|}
\hline Kidney Blood Press Res 2016;41:48-54 \\
\hline \begin{tabular}{l|l} 
DOI: $10.1159 / 000368546$ & $\begin{array}{l}\text { C } 2016 \text { S. Karger AG, Basel } \\
\text { www.karger.com/kbr }\end{array}$ \\
Published online: February 04, 2016 &
\end{tabular}
\end{tabular}

www.karger.com/kbr

Feng/Tang/Ye/Xue/Liao: Gene and Pathway Related to Kidney IR Injury

24 D'Ambrosio AL, Pinsky DJ, Connolly ES: The role of the complement cascade in ischemia/reperfusion injury: implications for neuroprotection. Mol Med 2001;7:367.

25 Pemberton M, Anderson G, Větvicka V, Justus D, Ross G: Microvascular effects of complement blockade with soluble recombinant CR1 on ischemia/reperfusion injury of skeletal muscle. J Immunol 1993;150:51045113.

26 Hill J, Lindsay T, Ortiz F, Yeh C, Hechtman H, Moore F: Soluble complement receptor type 1 ameliorates the local and remote organ injury after intestinal ischemia-reperfusion in the rat. J Immunol 1992;149:17231728.

27 Zamora MR, Davis R, Keshavjee S, Schulman L, Levin J, Ryan U, Patterson G: Complement inhibition attenuates human lung transplant reperfusion injury: a multicenter trial. CHEST J 1999;116:46S-46S.

28 Chávez-Cartaya RE, DeSola GP, Wright L, Jamieson NV, White DJ: Regulation of the complement cascade by soluble complement receptor type 1 . Protective effect in experimental liver ischemia and reperfusion. Transplantation 1995;59:1047-1052.

29 Weisman HF, Bartow T, Leppo MK, Marsh H, Carson GR, Concino MF, Boyle MP, Roux KH, Weisfeldt ML, Fearon DT: Soluble human complement receptor type 1: in vivo inhibitor of complement suppressing postischemic myocardial inflammation and necrosis. Science 1990;249:146-151.

30 Pinckard RN, O'Rourke RA, Crawford MH, Grover FS, McManus LM, Ghidoni JJ, Storrs SB, Olson MS: Complement localization and mediation of ischemic injury in baboon myocardium. J Clin Invest 1980;66:1050.

31 Weiss RH, Kim K: Metabolomics in the study of kidney diseases. Nat Rev Nephrol 2012;8:22-33.

32 Qingqing W, Xiao X, Paul F, Zheng D: Changes in metabolic profiles during acute kidney injury and recovery following ischemia/reperfusion. Plos One 2014;9:e106647.

33 Cadenas C, Vosbeck S, Hein EM, Hellwig B, Langer A, Hayen H, Franckenstein D, Büttner B, Hammad S, Marchan R: Glycerophospholipid profile in oncogene-induced senescence. Biochim Biophys Acta 2012;1821:1256-1268.

34 Goncalves G, Cenedeze M, Feitoza C, Wang P, Bertocchi A, Damiao M, Pinheiro H, Teixeira VA, Dos Reis M, Pacheco-Silva A: The role of heme oxygenase 1 in rapamycin-induced renal dysfunction after ischemia and reperfusion injury. Kidney Int 2006;70:1742-1749.

35 Nath KA, Balla G, Vercellotti GM, Balla J, Jacob HS, Levitt M, Rosenberg ME: Induction of heme oxygenase is a rapid, protective response in rhabdomyolysis in the rat. J Clin Invest 1992;90:267.

36 Jaeschke H, Woolbright BL: Current strategies to minimize hepatic ischemia-reperfusion injury by targeting reactive oxygen species. Transplant Rev 2012;26:103-114.

37 Takahashi T, Morita K, Akagi R, Sassa S: Protective role of heme oxygenase-1 in renal ischemia. Antioxid Redox Signal 2004;6:867-877.

38 Li L, Dong H, Song E, Xu X, Liu L, Song Y: Nrf2/ARE pathway activation, HO-1 and NQO1 induction by polychlorinated biphenyl quinone is associated with reactive oxygen species and PI3K/AKT signaling. Chem Biol Interact 2014;209:56-67.

39 Gang G-T, Hwang JH, Kim Y-H, Noh J-R, Kim K-S, Jeong JY, Choi DE, Lee KW, Jung J-Y, Shong M: Protection of NAD (P) H: quinone oxidoreductase 1 against renal ischemia/reperfusion injury in mice. Free Radic Biol Med 2014;67:139-149. 